\title{
Coupling electromagnetic pulse-shaped waves into wire-like interconnection structures with a non-linear protection - Time domain calculations by the PEEC method
}

\author{
G. Wollenberg and S. V. Kochetov \\ Otto-von-Guericke University Magdeburg, Institute for Fundamental Electrical Engineering and Electromagnetic \\ Compatibility, Universitätsplatz 2, D-39106 Magdeburg, Germany
}

\begin{abstract}
An interconnection system whose loads protected by a voltage suppressor and a low-pass filter against overvoltages caused by coupling pulse-shaped electromagnetic waves is analyzed. The external wave influencing the system is assumed as a plane wave with HPM form. The computation is provided by a full-wave PEEC model for the interconnection structure incorporated in the SPICE code. Thus, nonlinear elements of the protection circuit can be included in the calculation. The analysis shows intermodulation distortions and penetrations of low frequency interferences caused by intermodulations through the protection circuits. The example examined shows the necessity of using full-wave models for interconnections together with non-linear circuit solvers for simulation of noise immunity in systems protected by nonlinear devices.
\end{abstract}

\section{Introduction}

Modern technologies demand more importance for study of the intentional Electromagnetic Interferences (IEMI). Under IEMI we understand different types of electromagnetic environments that can be produced intentionally and can create operation problems (Radasky and Wik, 2003). There are two major categories of EM environments of concern: narrowband and wideband IEMI. Narrowband IEMI normally has a waveform that contents a frequency spectrum concentrated near a single frequency. Signals of this category can be also modulated sine waves or radio pulses. Repetitive series of modulated impulses make up a rather important class of narrowband IEMI. The repetitive series of modulated impulses or radio impulses have an important feature; they can produce the demodulation of low harmonics in the affected interconnection system that contains non-linear devices (Krauthäuser et al., 2002). Intermodulated oscillations produced in the equipment are quite dangerous because they

Correspondence to: G. Wollenberg

(wollenberg@et.uni-magdeburg.de) can occur at frequencies essentially lower then frequencies typically awaited from the incoming IEMI. The commercial equipment can not be protected from all frequencies especially in the relatively low frequency range and, consequently, it can be vulnerable for intermodulated oscillations. Typical technical concepts of equipments for protection from IEMI can be brought to design of filters and suppressors. Suppressors are non-linear devices that limit overvoltages. An effect of intermodulations caused by using a voltage suppressor is presented in this paper. Modeling intermodulations in arbitrary interconnection systems caused by an external EM field is a complex problem. A full-wave electromagnetic method is to be introduced for solution of this problem. This method has also to be able modeling non-linear effects. The PEEC method is a suitable approach for analysis considers the above explained problems (Ruehli, 1974). PEEC gives a possibility to create full-wave models of interconnection systems in the time and frequency domain non-linear elements of the circuit environment can be included (Wollenberg and Kochetov, 2002). The paper shows possibility that a fullwave PEEC solver integrated in SPICE (developed by our research group) is successfully applicable to problems of intermodulation modeling.

\section{Fundamentals of the PEEC method}

The PEEC method was developed by Albert E. Ruehli and is explained in detail in (Ruehli, 1974). It is based on the Electric Field Integral Equation (EFIE) and the Galerkin solution. Interconnections are to be divided into volume and surface cells. Surface cells are shifted from volume cells at one half of their length because of derivative approximation. Finally, this method gives us an equivalent chain circuit (called PEEC circuit). The PEEC circuit contains a chain of PEEC cells. The equivalent circuit of a PEEC cell is shown in Fig. 1, where $u_{k}^{i n c}$ is the voltage effected by the incident electromagnetic wave, $d_{k m}$ is the mean distance between partial element centers, $M_{L}$ is the set of volume cells, $M_{C}$ is the 


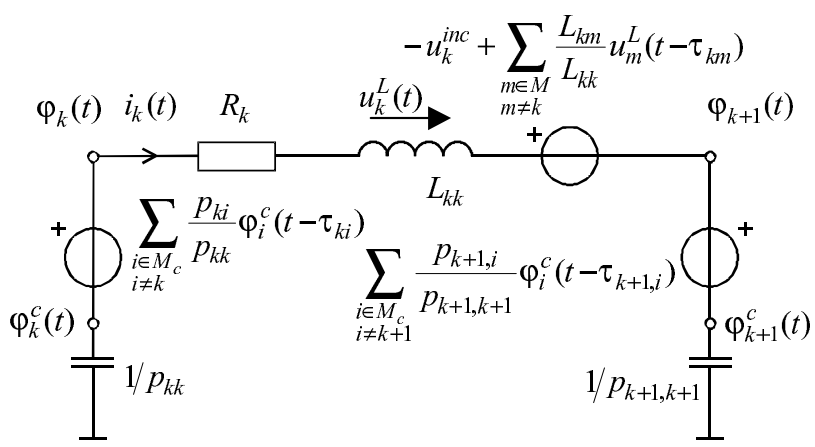

Fig. 1. Equivalent circuit model of the PEEC cell.

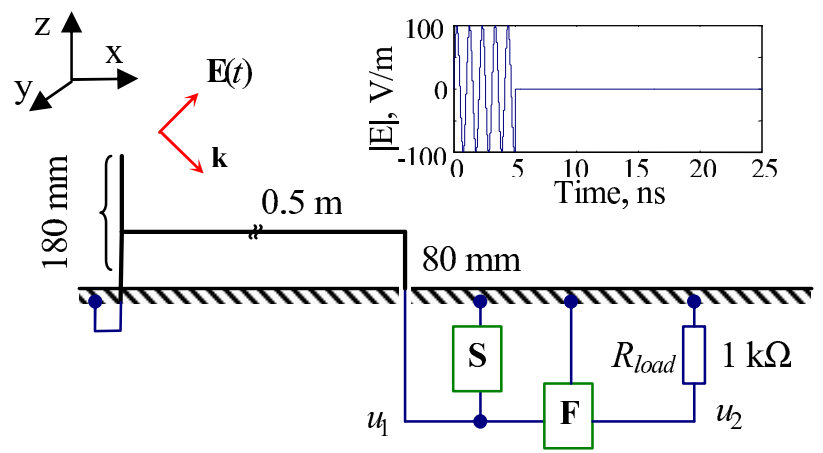

Fig. 2. The interconnection system protected by filter "F" and voltage suppressor "S".

set of surface cells, $L_{k m}$ and $p_{k m}$ are partial inductances and potential coefficients of the volume and surface cells, respectively (Ruehli, 1974). The partial elements are given by

$$
\begin{aligned}
& L_{k m}=\frac{\mu}{4 \pi a_{k} a_{m}} \cos \varphi_{k m} \int_{a_{k}} \int_{a_{m}^{\prime}} \int_{l_{k}} \int_{l_{m}^{\prime}} \frac{d a_{k} d a_{m}^{\prime} d l_{k} d l_{m}^{\prime}}{\left|\boldsymbol{r}-\boldsymbol{r}^{\prime}\right|}, \\
& p_{k i}=\frac{1}{4 \pi \epsilon_{0} S_{k} S_{i}} \int_{S_{k}} \int_{S_{i}^{\prime}} \frac{d S_{k} d S_{i}^{\prime}}{\left|\boldsymbol{r}-\boldsymbol{r}^{\prime}\right|},
\end{aligned}
$$

where $\cos \varphi_{k m}=\boldsymbol{e}_{k} \cdot \boldsymbol{e}_{m}$, with $\boldsymbol{e}_{k}, \boldsymbol{e}_{m}$ as unit vectors of the cell directions, $a_{k}$ and $a_{m}$ are cross-section areas of cells, $S_{k}$ and $S_{i}$ are surface areas, $l_{k}$ and $l_{m}$ are cell lengths.

The PEEC network of interconnections can be introduced into the circuit environment of a circuit solver. PEEC models were integrated in SPICE 3f4. Results obtained by this code are presented below.

\section{The problem setting}

A single transmission line connected with a short antenna is shown in Fig. 2. The left base of the line is loaded by a $10 \mathrm{k} \Omega$ resistor, the right base is grounded. The load is protected from the IEMI by a low-pass filter " $F$ " and a voltage suppressor "S". The interconnection system is disturbed by an external plane wave moving in the direction according to

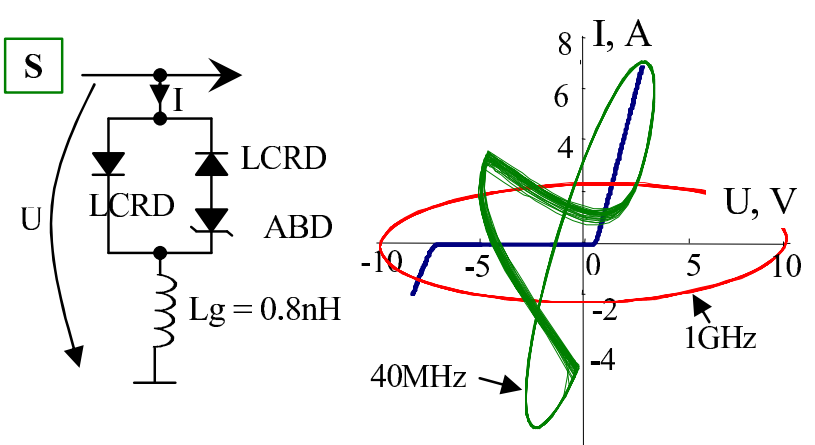

Fig. 3. Voltage suppressor SM16LC05 (Protek Devices), its static volt-amps diagram and its phase-plane portrait for frequencies $1 \mathrm{GHz}$ and $40 \mathrm{MHz}$.

vector $\boldsymbol{k}$ situated in the plane x, z (Fig. 2). The angle of incidence is $45^{\circ}$, the electric field vector is vertically polarized. The waveform of the excitation is a repetitive series of radio pulses with amplitude $100 \mathrm{~V}$ and a period of repetition of $25 \mathrm{~ns}$ and with the carrier frequency of $960 \mathrm{MHz}$. That corresponds to the frequency standard GSM(960 MHz/40 MHz). The problem is to compute the influence of this electromagnetic environment at the system under investigation and to appreciate the effectiveness of the protection devices.

The commercial semiconductor device SM16LC05 produced by Protek Devices is used as the voltage suppressor (Fig. 3). It is composed of two low capacitance rectifier diodes (LCRD) and an avalanche breakdown diode (ABD). The lead inductance $L_{g}$ is taken into account as well. A Spice model of the device is provided by the producer. The calculated by Spice static volt-amps diagram of the suppressor (Fig. 3) has a strongly asymmetrical character and an essential non-linearity. The phase-plane portraits of the suppressor computed by Spice for relatively high frequencies $40 \mathrm{MHz}$ and $1 \mathrm{GHz}$ show a complex dynamic dependency on the frequency and excitation amplitude as well. The protecting filter "F" is constructed as Sallen-Key second order low-pass circuit using operation amplifiers (Fig. 4). It is designed for an effective damping the signals with a frequency above $100 \mathrm{MHz}$. The frequency dependence of the voltage transfer function (Fig. 4) shows a $-3 \mathrm{~dB}$ damping for frequency of $51 \mathrm{MHz}$ and more then $-40 \mathrm{~dB}$ for the carrier frequency $960 \mathrm{MHz}$.

\section{Verification of PEEC results with MoM as a reference for linear loads}

Initially the system under investigation is simulated without protection. The results are computed by the PEEC method in the time domain and by MoM in the frequency domain with subsequent Inverse Fast Fourier Transformation (IFFT). The load voltage excited by the disturbing radio-pulse sequence is represented in Fig. 5 in two different time scales. Curves calculated by both methods correspond to each other 


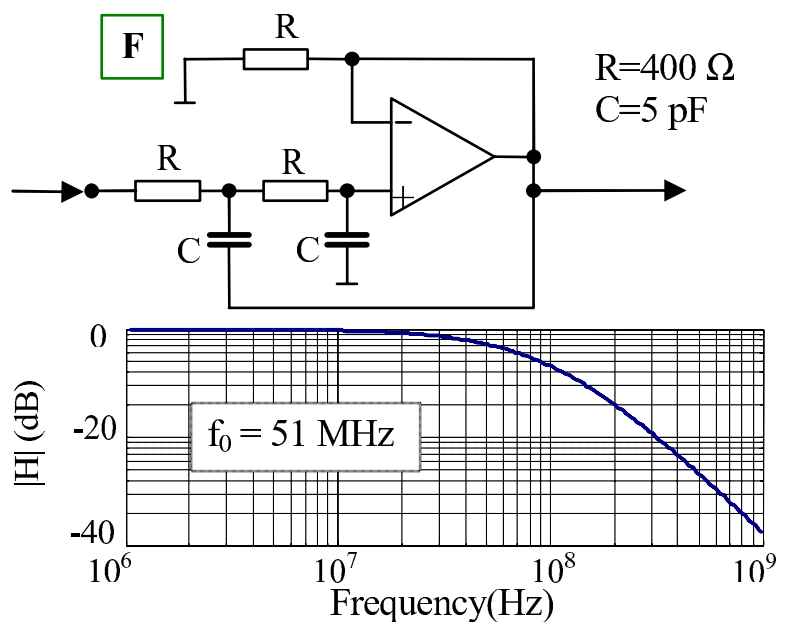

Fig. 4. Low-pass filter and its voltage transfer function.

with a good precision. The frequency spectrum of the disturbed load voltage is computed by FFT analysis (Fig. 6a). Obviously, the most of the spectrum power is situated near the carrier frequency $960 \mathrm{MHz}$ and its satellites. However, the eigenfrequency of the transmission line (about $110 \mathrm{MHz}$ ) and the modulation frequency $40 \mathrm{MHz}$ can be also noticed. It can be expected that the protecting filter " $F$ " will damp the excited signal quite effective but it is difficult to predict the non-linear distortion of the spectrum produced by the suppressor.

\section{Results obtained for the protected load}

At first the voltage suppressor is added in the system without filter. A simulation by the PEEC method gives us a transient curve of the load voltage (Fig. 7b). Obviously, the spectrum of the voltage is essentially changed in comparison with the result computed for linear load (Fig. 5). The low harmonics with a frequency about $40 \mathrm{MHz}$ are visible. The FFT spectrum analysis (Fig. 6c) shows us following changes. The carrier frequency is attenuated approximately by 100 times. The frequency of modulation $40 \mathrm{MHz}$, however, amplified by $120 \%$. The inclusion of the suppressor in parallel to the $10 \mathrm{k} \Omega$ load can only diminish the level of the load voltage if we have not a demodulation effect. Thus, we can conclude that the demodulation takes place. The second significant frequency of the spectrum is about $220 \mathrm{MHz}$. This frequency corresponds to the first voltage magnitude maximum in a transmission line loaded by a resistor being much less then the wave impedance. Therefore, we conclude that the suppressor reduces the $10 k \Omega$ impedance essentially, so that the character of wave reflections in the interconnection system is changed. The eigenfrequency of the interconnection system for the configuration without suppressor about $110 \mathrm{MHz}$ (Fig. 6a) is disappeared, because with a little load we have a minimum of the voltage magnitude at this frequency. The next step is to add in the configuration the low-

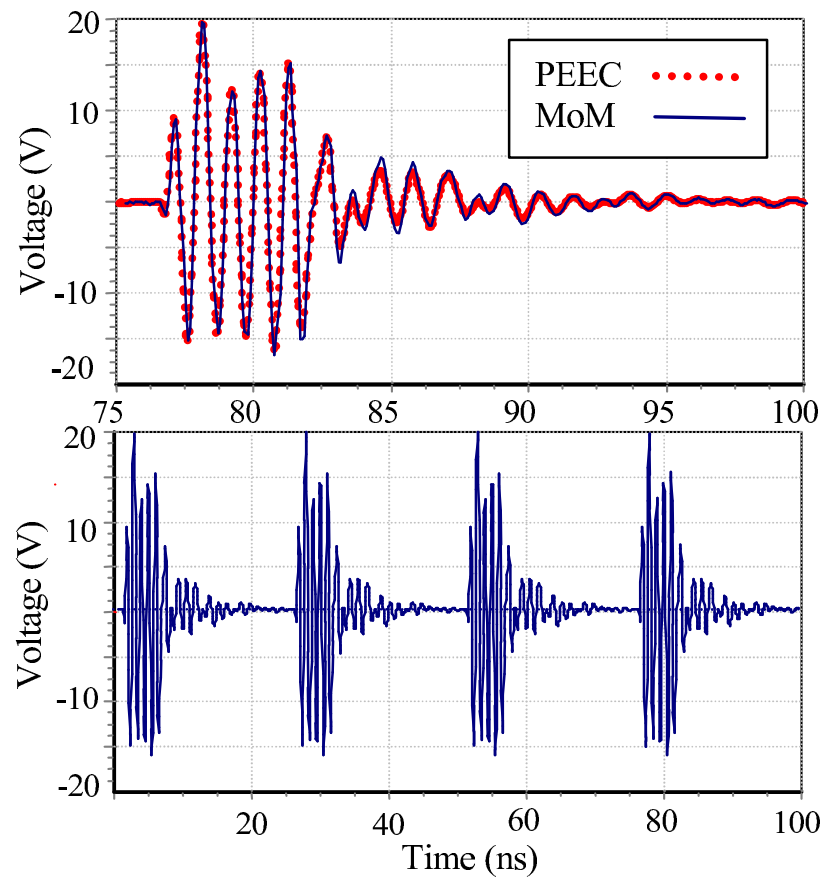

Fig. 5. The load voltage calculated by PEEC and MoM (FD-IFFT), calculation provided without the filter and the suppressor.

pass filter based on operational amplifier and to investigate the system with and without the voltage suppressor. The time response of the load voltage computed for the system protected only by the filter (Fig. 7c) shows big changes in comparison with the curve from Fig. 5. Obviously, the high frequency harmonics are damped what is confirmed by the spectrum analysis (Fig. 6b). The amplitudes of harmonics lower then $100 \mathrm{MHz}$ are conserved but all higher frequencies are damped according to the frequency response of the following transfer function calculated in the frequency domain:

$T(j \omega)=U_{2}(j \omega) / E(j \omega)$

where $E$ is the incident field, $U_{2}$ is the load voltage. The frequency responses for the transfer function are obtained for the system with and without filter (Fig. 8). Obviously, the interconnection system loaded only by a resistor has a first resonance at $110 \mathrm{MHz}$ where it absorbs most of energy. Including of the filter with a big input impedance does not change essentially the frequency response of the voltage at the filter input $U_{1}$ (Fig. 2) and provides an essential damping. for frequencies higher then $100 \mathrm{MHz}$ in load voltage $U_{2}$. The last configuration is protected by the voltage suppressor and the filter. The time curve of the load voltage (Fig. 7a) looks like a sine of $40 \mathrm{MHz}$ with distortions. The spectrum analysis of the interference (Fig. 6d) shows that the high frequency part of the spectrum near the carrier frequency is completely suppressed. In the same time the modulation frequency amplitude increases to $230 \mathrm{mV}$. Obviously, we see the effect of demodulation caused by the non-linear properties of the suppressor and selected from spectrum via the filter. The 


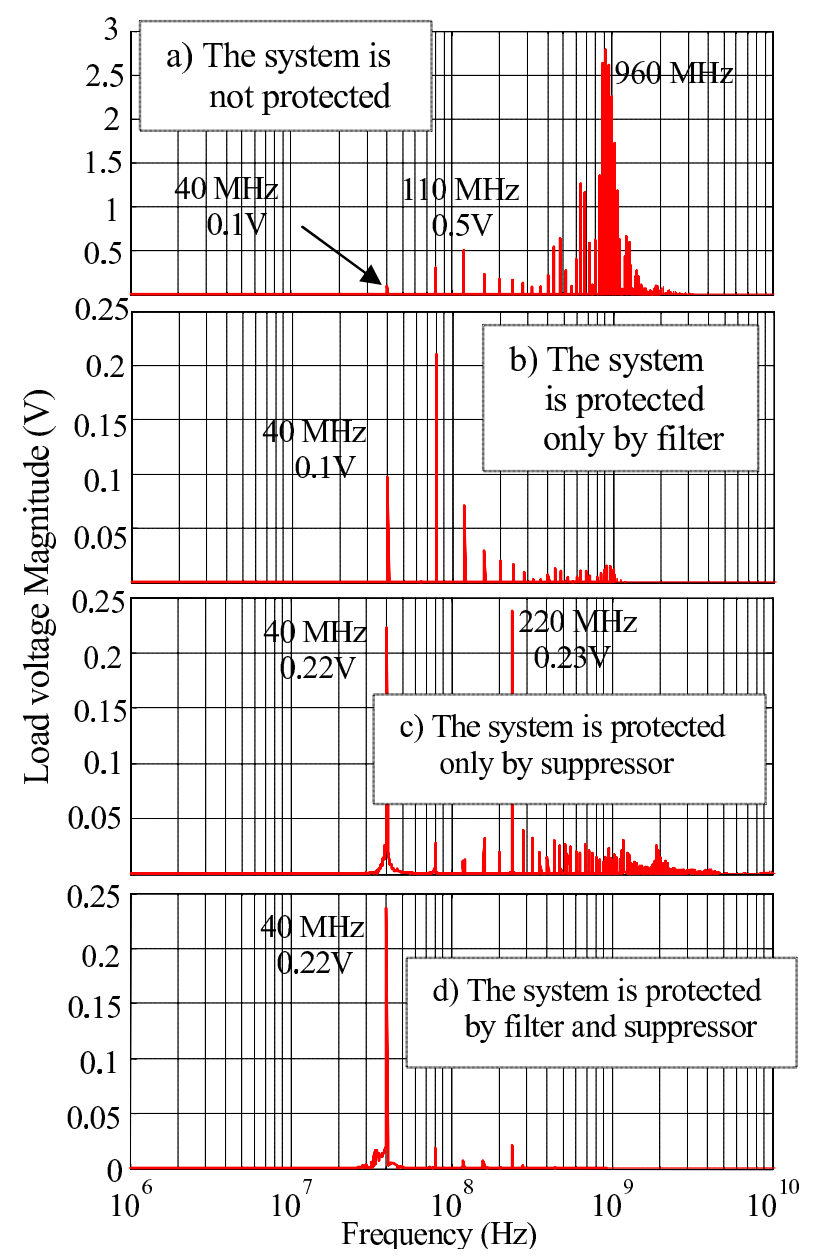

Fig. 6. The spectrum magnitude of the load voltage for different protection measures.

eigenfrequency of the interconnection system of $220 \mathrm{MHz}$ can be noticed as strongly damped by the filter in comparison to Fig. 6c. Thus, the frequency spectrum of the disturbance is completely transformed to low frequency oscillations. The intermodulations have produced also frequencies lower than the modulation frequency of $40 \mathrm{MHz}$ which does not play a significant part in the signal.

\section{Conclusions}

An instance of an interconnection system with a lumped load protected by a semiconductor voltage suppressor and a lowpass filter being disturbed by a series of radio pulses is investigated using a code based on PEEC models incorporated in SPICE. For the propose of verification, the considered interconnection system in the same electromagnetic environment but without non-linear loads was modeled by another code based on MoM. Results obtained by MoM and PEEC are completely the same. A time domain analysis of interferences is provided by the combination of a full-wave PEEC solver with the analysis of intermodulations caused by a suf-

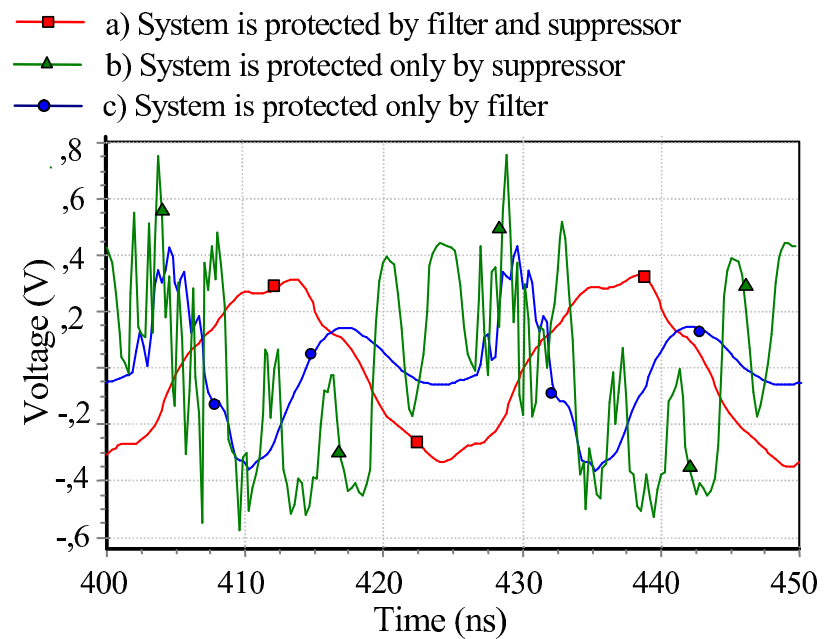

Fig. 7. The load voltage in the protected system.

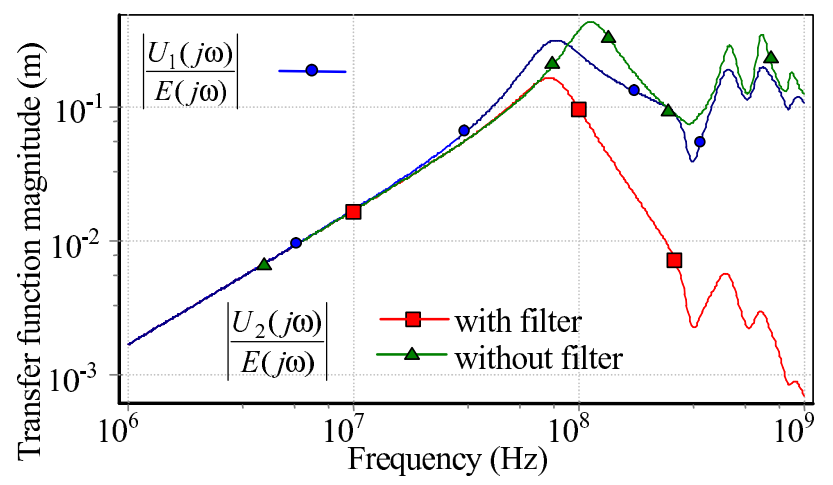

Fig. 8. Transfer function magnitude.

ficiently non-linear load. The results show the effect of the frequency demodulation and the increase of low frequency disturbances on the load produced by the non-linear protection.

Acknowledgements. This study has been carried out with financial support from the Deutsche Forschungsgemeinschaft.

\section{References}

Krauthäuser, H. G., Tkachenko, S., and Nitsch, J.: The action of non-linear effect in a resonator, XXVIIth General Assembly of the International Union of Radio Science, 2167, 2002.

Radasky, W. A. and Wik, M. W.: Intentional (IEMI) - Understanding the threat and developing protection concepts, 15th International Zurich Symposium and Technical Exhibition on EMC, 111-114, 2003.

Ruehli, A. E.: Equivalent circuit models for three-dimensional multiconductor systems, IEEE Trans. Microwave Theory Techn., Vol. 22, 216-221, 1974.

Wollenberg, G. and Kochetov, S. V.: Coupling into 3D interconnection structures with nonlinear loads - an application of the method of partial elements, XXVIIth General Assembly of the International Union of Radio Science, E8-O.6, 2002. 